\title{
Robust Fault Detection for a Class of Uncertain Nonlinear Systems Based on Multiobjective Optimization
}

\author{
Bingyong Yan, Huifeng Wang, and Huazhong Wang \\ Key Laboratory of Advanced Control and Optimization for Chemical Process of Ministry of Education, \\ Department of Automation, East China University of Science and Technology, Shanghai 200237, China
}

Correspondence should be addressed to Huazhong Wang; hzwang@ecust.edu.cn

Received 30 August 2014; Accepted 12 October 2014

Academic Editor: Wei Zhang

Copyright (C) 2015 Bingyong Yan et al. This is an open access article distributed under the Creative Commons Attribution License, which permits unrestricted use, distribution, and reproduction in any medium, provided the original work is properly cited.

A robust fault detection scheme for a class of nonlinear systems with uncertainty is proposed. The proposed approach utilizes robust control theory and parameter optimization algorithm to design the gain matrix of fault tracking approximator (FTA) for fault detection. The gain matrix of FTA is designed to minimize the effects of system uncertainty on residual signals while maximizing the effects of system faults on residual signals. The design of the gain matrix of FTA takes into account the robustness of residual signals to system uncertainty and sensitivity of residual signals to system faults simultaneously, which leads to a multiobjective optimization problem. Then, the detectability of system faults is rigorously analyzed by investigating the threshold of residual signals. Finally, simulation results are provided to show the validity and applicability of the proposed approach.

\section{Introduction}

In recent years, with the development of intelligent control technology and processing ability of microchips, modern dynamic systems are becoming more and more complex. Fault detection and identification (FDI) can be deployed for monitoring and reacting to the faults occurring in these modern dynamic systems, which has a broad range of applications including intelligent power grids, underwater robot, high voltage direct current transmission lines, and long transmission lines in pneumatic, chemical processes. Effective FDI schemes can ensure safety and reliability of complex dynamic systems. The most fundamental problem for FDI is to develop a fault diagnosis observer or fault diagnosis filter. Due to the development of various nonlinear or linear states observers, the model-based analytical redundancy approaches received more and more attention in the last two decades, including observer based method, parity space based method, eigenstructure assignment based method, and parameter identification based method and $H_{\infty}$ filter based method [1-8].

Early fault diagnosis approaches often assumed the availability of an accurate dynamic system model. In practice, however, such an assumption can be invalid. The main reason is that unstructured modeling uncertainties are always unavoidable when modeling system mathematical structures. Therefore, there are a growing number of researchers focusing their interests on FDI for nonlinear systems with uncertainty. A novel integrated fault diagnosis and fault tolerant control algorithm for non-Gaussian singular timedelayed stochastic distribution control system was proposed based on iterative learning observer. The iterative learning observer was developed to obtain estimation of system faults. Recently in [9], the authors introduced a novel fault detection and diagnosis for nonlinear non-Gaussian dynamic processes using kernel dynamic independent component analysis method. Sensor fault diagnosis and identification in nonlinear plants were discussed in [10]. The faults under consideration in [10] were assumed to be abruptly occurring calibration errors. Thus, an adaptive particle filter was developed to diagnose sensor faults and compensate for their effects. Chen and Saif in [11] proposed an iterative learning observer (ILO) based fault diagnosis approach for fault detection, identification, and accommodation. The main characteristic of the ILO was that its states were updated or driven successively by the estimation errors of previous system outputs and control inputs. The observer gain matrix and adaptive adjusting rule of the fault estimator are investigated in detail. 
In our previous work [12], a robust fault tracking approximator (RFTA) based fault detection and identification scheme for a class of nonlinear systems was developed and its stability properties were investigated as well. Due to the existence of unstructured modeling uncertainties, the convergence speed and fault tracking accuracy of the FTA will be influenced dramatically. The objective of this work is to extend the previous research results to a class of nonlinear systems with uncertainty and optimize the gain matrix of FTA. First of all, we decompose the fault diagnosis problem into a parameter optimization problem and a fault detection problem. The parameter optimization problem can be described as follows: by using robust control technology and parameter optimization algorithms, the gain matrix of FTA is designed to minimize the effects of system uncertainty on residual signals while maximizing the effects of system faults on residual signals. The design of the gain matrix of FTA takes into account the robustness to system uncertainty and sensitivity to system faults simultaneously, which leads to a multiobjective optimization problem. The fault detection problem can be described as follows: we detect the system faults according to the relationship between the calculated threshold and residual signals. The detectability of system faults is rigorously analyzed by investigating the threshold of residual signals. In the end, an illustrative example is proposed to demonstrate the validity and applicability of the proposed approach.

An outline of this paper is organized as follows. In Section 2, we define a class of uncertain nonlinear systems and present a multiobjective optimization problem. The design of gain matrix of FTA is investigated in Section 3. The calculation of threshold for fault detection is designed in Section 4. In Section 5, simulation results are reported illustrating the effectiveness of the proposed robust fault detection scheme. Some conclusion remarks are provided in Section 6 .

\section{Problem Formulation}

Consider a class of uncertain nonlinear systems described by

$$
\dot{x}(t)=A x(t)+B u(t)+g(x(t), t)+B_{f} f(t)+B_{d} d(t),
$$

$$
y(t)=C x(t)+D u(t)+D_{f} f(t)+D_{d} d(t),
$$

where $x(t) \in R^{n}$ is the system state vector, $u(t) \in R^{p}$ is the control input vector, $y(t) \in R^{q}$ is the measurement output vector, $d(t)$ is the system uncertainty that belongs to $L_{2}^{m}[0,+\infty], f(t) \in R^{m}$ is the system fault to be detected, and $g(\cdot)$ : is a known nonlinear function. $A, B, B_{f}, B_{d}, C, D$, $D_{f}$, and $D_{d}$ are known parameter matrix with appropriate dimensions. We take the following assumptions.

Assumption 1. The observability matrix associated with the pair $(A, C)$ is full rank.

Assumption 2. The function $d(t)$ in (1), representing the unstructured modeling uncertainty, is bounded by a known constant; that is, $\|d(t)\| \leq L_{w}$.
Assumption 3. The nonlinear function $g(\cdot)$ satisfies Lipschitz condition; that is,

$$
\begin{array}{r}
\|g(x(t), t)-g(y(t), t)\| \leq\|\rho(x(t)-y(t))\| \\
\forall x(t), y(t) \in D,
\end{array}
$$

where $\rho$ is a known real constant. Throughout the paper, the notation $\|\cdot\|$ will be used to denote the Euclidean norm of a vector.

To detect a fault, the FTA is constructed as follows [12]:

$$
\begin{gathered}
\dot{\hat{x}}_{k}=A \widehat{x}_{k}(t)+g\left(\widehat{x}_{k}(t), t\right)+B u_{k}(t)+B \widehat{f}_{k}(t) \\
+H(y(t)-\widehat{y}(t)) \\
\widehat{y}_{k}(t)=C \widehat{x}_{k}(t)+D u_{k}(t), \\
e_{k}(t)=x_{k}(t)-\widehat{x}_{k}(t), \\
r_{k}(t)=C e_{k}(t), \\
\widehat{f}_{k+1}(t)=\widehat{f}_{k}(t)+\Gamma \dot{r}_{k}(t), \\
\left\|y_{k}(t)-\widehat{y}_{k}(t)\right\|_{\infty} \leq \gamma, \quad t \in\left[t_{a}, t_{b}\right],
\end{gathered}
$$

where $\widehat{x}_{k}(t) \in R^{n}, \widehat{y}_{k}(t) \in R^{q}$ are estimated system state and output, respectively. $k$ is the iteration index and $\gamma$ is the given performance index. $\Gamma$ is constant gain matrix, and its elements are within the scope $(0,1) . \widehat{f}_{k}(t)$ is virtual fault, which is an estimate of $f(t)$ and the value of $\widehat{f}(t)$ is set to zero until a fault is detected. $e(t)$ denotes the system state estimation error. $e_{k}(t)$ denotes the estimation error of $e(t)$ after $k$ th iterative operation. $r_{k}(t)$ denotes the estimation error of $r(t)$ after $k$ th iterative operation. $H$ is the gain matrix to be optimized. $r(t)$ is the so-called generated residual signal.

The basic idea behind the FTA is to adjust the virtual fault $\widehat{f}_{k}(t)$ within a specified time horizon by using iterative learning algorithm such that the virtual fault can approximate the system fault $f(t)$ as closely as possible. Detailed description about the FTA can be found in [12].

From (4) we can clearly see that the value of gain matrix $H$ will have a great influence on the convergence speed of FTA. As a result, the fault tracking accuracy will be influenced as well. In previous work [12], we have investigated the stability and fault tracking accuracy of the FTA on the assumption that the gain matrix is a prespecified value. However, the design of gain matrix $H$ still remains unresolved. In this work, we will utilize multiobjective parameter optimization algorithm and robust control theory to optimize the gain matrix $H$.

\section{Design of Gain Matrix}

First, we convert the parameter optimization problem into a performance index optimization problem. Then, the design of gain matrix is given in terms of linear matrix inequality (LMI). 
Define the state estimation error $e(t)=x(t)-\widehat{x}(t)$, and then it follows from (1)-(7) that [13-17]

$$
\begin{aligned}
\dot{e}(t)= & (A-H C) e(t)+\left(B_{f}-H D_{f}\right) f(t) \\
& +\left(B_{d}-H D_{d}\right) d(t)+g(x(t), t)-g(\widehat{x}(t), t), \\
r(t)= & C e(t)+D_{f} f(t)+D_{d} d(t),
\end{aligned}
$$

where gain matrix $H$ is to be designed such that the system (10) is asymptotically stable. To optimize the gain matrix $H$, we propose the following performance index [18]:

$$
J=\frac{\left\|T_{r d}\right\|_{\infty}}{\left\|T_{r f}\right\|_{-}}
$$

where $T_{r d}$ denotes the transfer function from system uncertainty to residual signal and $T_{r f}$ denotes the transfer function from system faults to residual signal. The objective of this parameter optimization problem is to maximize the effects of system faults on residual signals while minimizing the effects of system uncertainty on residual signals. Thus, it leads to the following optimization problem: finding a gain matrix $H$, such that the system (10) remains asymptotically stable, and the performance index $J=\left\|T_{r d}\right\|_{\infty} /\left\|T_{r f}\right\|_{-}$is minimized.

Remark 4. The item $\left\|T_{r d}\right\|_{\infty}$ is used to measure the robustness of residual signals to system uncertainty, while the sensitivity of residual signals to system faults is measured by $\left\|T_{r f}\right\|_{-}=\inf _{f \in(0, \infty)}\left[T_{r f}(j w)\right]$ and $\underline{\sigma}\left[T_{r f}(j w)\right]$ denotes the nonzero singular value of $T_{r f}(j w)$. The optimization problem described by (11) is actually a multiobjective optimization problem, and it can be formulated as follows: for a given constant $\gamma>0, \beta>0$, finding a gain matrix $H$, such that the system (10) remains asymptotically stable, and satisfying the following inequality [18]:

$$
\|r(t)\|_{\infty} \leq \gamma\|d(t)\|_{\infty}, \quad\|r(t)\|_{-}>\beta\|f(t)\|_{-}
$$

with (10); note that the dynamics of the residual signal depends not only on $f(t)$ and $d(t)$, but also on the nonlinear part: $g(x(t), t)-g(\widehat{x}(t), t)$. So the traditional fault detection observer design methods cannot be used here. A novel method to design gain matrix $H$, meeting the performance index (12), is required. In this paper, we propose a method to resolve this problem in terms of LMIs.

Lemma 5 (see $[18,19])$. Let $A$ and $B$ be real matrices with appropriate dimensions. For any scalar $\varepsilon>0$ and vectors $x, y \in R^{n}$, then

$$
2 x^{T} A B y \leq \varepsilon^{-1} x^{T} A A^{T} x+\varepsilon y^{T} B^{T} B y .
$$

Theorem 6. Given a constant $\gamma>0$, in the condition of $f(t)=0$, the system (10) is asymptotically stable and satisfies $\|r(t)\|_{\infty} \leq \gamma\|d(t)\|_{\infty}$, if there exists a positive symmetrical matrix $P$, scalar $\varepsilon_{1}>0$, satisfying the following LMI:

$$
\left[\begin{array}{ccc}
M_{1} & M_{3} & P \\
M_{3}^{T} & M_{2} & 0 \\
P^{T} & 0 & -\varepsilon_{1} I
\end{array}\right]<0,
$$

where

$$
\begin{aligned}
& M_{1}=P(A-H C)+(A-H C)^{T} P+C^{T} C+\varepsilon_{1} \rho^{2} I, \\
& M_{2}=D_{d}^{T} D_{d}-\gamma^{2} I, \\
& M_{3}=C^{T} D_{d}+P\left(B_{d}-H D_{d}\right) .
\end{aligned}
$$

Proof. We choose a Lyapunov function of the form:

$$
V(t)=e^{T}(t) P e(t),
$$

where $P$ is a positive symmetrical matrix. In the fault-free case, when the system uncertainty $d(t)=0$, we have

$$
\begin{aligned}
\dot{V}(t)= & e^{T}(t) P \dot{e}(t)+\dot{e}^{T}(t) P e(t) \\
= & e^{T}(t) P[(A-H C) e(t)+g(x(t), t)-g(\widehat{x}(t), t)] \\
& +[(A-H C) e(t)+g(x(t), t)-g(\widehat{x}(t), t)]^{T} P e \\
= & e^{T}(t)\left[P(A-H C)+(A-H C)^{T} P\right] e(t) \\
& +e^{T}(t) P[g(x(t), t)-g(\widehat{x}(t), t)] \\
& +[g(x(t), t)-g(\widehat{x}(t), t)]^{T} e P .
\end{aligned}
$$

According to Lemma 5 and Assumption 3, we have

$$
\begin{aligned}
2 e^{T}(t) P[g(x(t), t)-g(\widehat{x}(t), t)] \\
\leq \varepsilon_{1}^{-1} e^{T}(t) P P^{T} e(t)+\varepsilon_{1}(g(x(t), t)-g(\widehat{x}(t), t))^{T} \\
\quad \times(g(x(t), t)-g(\widehat{x}(t), t)) \\
\leq \varepsilon_{1}^{-1} e^{T}(t) P P^{T} e(t)+\varepsilon_{1} \rho^{2} e^{T}(t) e(t) .
\end{aligned}
$$

Thus:

$$
\begin{gathered}
\dot{V}(t) \leq e^{T}(t)\left\lfloor P(A-H C)+(A-H C)^{T} P\right. \\
\left.+\varepsilon_{1}^{-1} P P^{T}+\varepsilon_{1} \rho^{2} I\right\rfloor e(t) .
\end{gathered}
$$

According to (14), we can obtain that $\dot{V}(t) \leq 0$. So the system (10) is asymptotically stable in the condition of no fault.

When the system uncertainty $d(t) \neq 0$, define

$$
H(e, d)=\dot{V}(t)+\|r(t)\|_{\infty}-\gamma^{2}\|d(t)\|_{\infty} .
$$

So we have

$$
\begin{aligned}
H(e, d)= & e^{T}(t) P \dot{e}(t)+\dot{e}^{T}(t) P e(t)+e^{T}(t) C^{T} C e(t) \\
& +e^{T}(t) C^{T} D_{d} d(t)+d^{T}(t) D_{d}^{T} C e(t) \\
& +d^{T}(t) D_{d}^{T} D_{d} d(t)-\gamma^{2} d^{T}(t) d(t) \\
= & e^{T}(t) P\left[(A-H C) e(t)+\left(B_{d}-H D_{d}\right) d(t)\right. \\
& \quad+g(x(t), t)-g(\widehat{x}(t), t)] \\
& +[(A-H C) e(t)+g(x(t), t)-g(\widehat{x}(t), t) \\
& \left.\quad+\left(B_{d}-H D_{d}\right) d(t)\right]^{T} P e(t)
\end{aligned}
$$




$$
\begin{aligned}
& +e^{T}(t) C^{T} C e(t)+e^{T}(t) C^{T} D_{d} d(t) \\
& +d^{T}(t) D_{d}^{T} C e(t)+d^{T}(t) D_{d}^{T} D_{d} d(t) \\
& -\gamma^{2} d^{T}(t) d(t) \\
\leq & e^{T}(t)\left[P(A-H C)+(A-H C)^{T} P+\varepsilon_{1}^{-1} P P^{T}\right. \\
& \left.+C^{T} C+\varepsilon_{1} \rho^{2} I\right] e(t)+e^{T}(t)\left[C^{T} D_{d}\right] d(t) \\
& +d^{T}(t)\left[D_{d}^{T} C\right] e(t)+d^{T}(t) D_{d}^{T} D_{d} d(t) \\
& -\gamma^{2} d^{T}(t) d(t)+e^{T}(t)\left[P\left(B_{d}-H D_{d}\right)\right] d(t) \\
& +d^{T}(t)\left[\left(B_{d}-H D_{d}\right)^{T} P\right] e(t) \\
= & {\left[\begin{array}{l}
e(t) \\
d(t)
\end{array}\right]^{T}\left[\begin{array}{ll}
M_{1} & M_{3} \\
M_{3}^{T} & M_{2}
\end{array}\right]\left[\begin{array}{l}
e(t) \\
d(t)
\end{array}\right], }
\end{aligned}
$$

where

$$
\begin{aligned}
& M_{1}=P(A-H C)+(A-H C)^{T} P+C^{T} C+\varepsilon_{1}^{-1} P P^{T}+\varepsilon_{1} \rho^{2} I, \\
& M_{2}=D_{d}^{T} D_{d}-\gamma^{2} I, \\
& M_{3}=C^{T} D_{d}+P\left(B_{d}-H D_{d}\right) .
\end{aligned}
$$

According to (14) and Schur theory, we can obtain that

$$
H(e, d)=\dot{V}(t)+r^{T}(t) r(t)-\gamma^{2} d^{T}(t) d(t)<0 .
$$

For any given time $t>0$, integration of (23) from 0 to $t$ yields

$$
\int_{0}^{+\infty} r^{T}(t) r(t)<\gamma^{2} \int_{0}^{+\infty} d^{T}(t) d(t)
$$

Thus, the inequality $\|r(t)\|_{\infty} \leq \gamma\|d(t)\|_{\infty}$ holds. This completes the proof.

Theorem 7. Given a constant $\beta>0$, in the condition of $d(t)=$ 0 , the system (10) is asymptotically stable and satisfies $\|r(t)\|_{-} \geq$ $\beta\|f(t)\|_{-}$, if there exists a positive symmetrical matrix $Q$, scalar $\eta_{1}>0$, satisfying the following LMI:

$$
\left[\begin{array}{ccc}
N_{1} & N_{3} & Q \\
N_{3}^{T} & N_{2} & 0 \\
Q^{T} & 0 & -\eta_{1} I
\end{array}\right]<0,
$$

where

$$
\begin{aligned}
& N_{1}=Q(A-H C)+(A-H C)^{T} Q-C^{T} C+\eta_{1} \rho^{2} I, \\
& N_{2}=-D_{f}^{T} D_{f}+\beta^{2} I, \\
& N_{3}=-C^{T} D_{f}+Q\left(B_{f}-H D_{f}\right) .
\end{aligned}
$$

Proof. We choose a Lyapunov function of the form:

$$
V(t)=e^{T}(t) Q e(t),
$$

where $Q$ is a positive symmetrical matrix. In the condition of $d(t)=0$, when the system faults $f(t)=0$, we have

$$
\begin{aligned}
\dot{V}(t)= & e^{T}(t) Q \dot{e}(t)+\dot{e}^{T}(t) Q e(t) \\
= & e^{T}(t) Q[(A-H C) e(t)+g(x(t), t)-g(\widehat{x}(t), t)] \\
& +[(A-H C) e(t)+g(x(t), t)-g(\widehat{x}(t), t)]^{T} Q e \\
= & e^{T}(t)\left[Q(A-H C)+(A-H C)^{T} Q\right] e(t) \\
& +e^{T}(t) Q[g(x(t), t)-g(\widehat{x}(t), t)] \\
& +[g(x(t), t)-g(\widehat{x}(t), t)]^{T} e Q .
\end{aligned}
$$

According to Lemma 5 and Assumption 3, we have

$$
\begin{aligned}
2 e^{T}(t) & Q[g(x(t), t)-g(\widehat{x}(t), t)] \\
\leq & \eta_{1}^{-1} e^{T}(t) Q Q^{T} e(t)+\eta_{1}(g(x(t), t)-g(\widehat{x}(t), t))^{T} \\
& \times(g(x(t), t)-g(\widehat{x}(t), t)) \\
\leq & \eta_{1}^{-1} e^{T}(t) Q Q^{T} e(t)+\eta_{1} \rho^{2} e^{T}(t) e(t) .
\end{aligned}
$$

Thus

$$
\begin{aligned}
\dot{V}(t) \leq e^{T}(t)[Q & (A-H C)+(A-H C)^{T} Q \\
& \left.+\eta_{1}^{-1} P P^{T}+\eta_{1} \rho^{2} I\right] e(t) .
\end{aligned}
$$

According to (25), we can obtain that $\dot{V}(t) \leq 0$. So the system (10) is asymptotically stable in the condition of $f(t)=0$.

When the system faults $f(t) \neq 0$, define

$$
H(e, f)=\dot{V}(t)+\beta^{2}\|f(t)\|_{\infty}-\|r(t)\|_{\infty} .
$$

So we have

$$
\begin{aligned}
H(e, f)= & e^{T}(t) Q \dot{e}(t)+\dot{e}^{T}(t) Q e(t)-e^{T}(t) C^{T} C e(t) \\
& -e^{T}(t) C^{T} D_{f} f(t)-f^{T}(t) D_{f}^{T} C e(t) \\
& -f^{T}(t) D_{f}^{T} D_{f} f(t)+\beta^{2} f^{T}(t) f(t) \\
= & e^{T}(t) Q\left[(A-H C) e(t)+\left(B_{f}-H D_{f}\right) f(t)\right. \\
& +g(x(t), t)-g(\widehat{x}(t), t)] \\
& +[(A-H C) e(t)+g(x(t), t)-g(\widehat{x}(t), t) \\
& \left.+\left(B_{f}-H D_{f}\right) f(t)\right]^{T} Q e(t)-e^{T}(t) C^{T} C e(t) \\
& -e^{T}(t) C^{T} D_{f} d(t)-f^{T}(t) D_{f}^{T} C e(t) \\
& -f^{T}(t) D_{f}^{T} D_{f} f(t)+\beta^{2} f^{T}(t) f(t) \\
\leq & e^{T}(t)\left[Q(A-H C)+(A-H C)^{T} Q+\eta_{1}^{-1} Q Q^{T}\right. \\
& \left.\quad-C^{T} C+\eta_{1} \rho^{2} I\right] e(t)-e^{T}(t)\left[C^{T} D_{f}\right] f(t)
\end{aligned}
$$




$$
\begin{aligned}
& -f^{T}(t)\left[D_{f}^{T} C\right] e(t)-f^{T}(t) D_{f}^{T} D_{f} f(t) \\
& +\beta^{2} f^{T}(t) f(t)-e^{T}(t)\left[Q\left(B_{d}-H D_{d}\right)\right] f(t) \\
& -f^{T}(t)\left[\left(B_{f}-H D_{f}\right)^{T} Q\right] e(t) \\
& =\left[\begin{array}{c}
e(t) \\
f(t)
\end{array}\right]^{T}\left[\begin{array}{ll}
N_{1} & N_{3} \\
N_{3}^{T} & N_{2}
\end{array}\right]\left[\begin{array}{l}
e(t) \\
f(t)
\end{array}\right],
\end{aligned}
$$

where

$$
\begin{aligned}
N_{1}= & Q(A-H C)+(A-H C)^{T} Q-C^{T} C \\
& +\eta_{1}^{-1} Q Q^{T}+\eta_{1} \rho^{2} I \\
N_{2}= & -D_{f}^{T} D_{f}+\beta^{2} I \\
N_{3}= & -C^{T} D_{f}+Q\left(B_{f}-H D_{f}\right) .
\end{aligned}
$$

According to Schur theory, we can obtain that

$$
H(e, f)=\dot{V}(t)+\beta^{2} f^{T}(t) f(t)-r^{T}(t) r(t)<0 .
$$

For any given time $t>0$, integration of (34) from 0 to $t$ yields

$$
\int_{0}^{+\infty} r^{T}(t) r(t)>\beta^{2} \int_{0}^{+\infty} f^{T}(t) f(t) .
$$

Thus, the inequality $\|r(t)\|_{-}>\beta\|f(t)\|_{-}$holds. This completes the proof.

Theorem 8. Given constants $\gamma>0$ and $\beta>0$, the system (10) is asymptotically stable and satisfies $\|r(t)\|_{\infty} \leq \gamma\|d(t)\|_{\infty}$ and $\|r(t)\|_{-} \geq \beta\|f(t)\|_{-}$, if there exist matrices $P, Q$, and $H$, scalar $\varepsilon_{1}>0, \eta_{1}>0$ satisfying matrix inequality:

$$
\left[\begin{array}{ccc}
M_{1} & M_{3} & P \\
M_{3}^{T} & M_{2} & 0 \\
P^{T} & 0 & -\varepsilon_{1} I
\end{array}\right]<0, \quad\left[\begin{array}{ccc}
N_{1} & N_{3} & Q \\
N_{3}^{T} & N_{2} & 0 \\
Q^{T} & 0 & -\eta_{1} I
\end{array}\right]<0,
$$

where

$$
\begin{aligned}
& M_{1}=P(A-H C)+(A-H C)^{T} P+C^{T} C+\varepsilon_{1} \rho^{2} I, \\
& M_{2}=D_{d}^{T} D_{d}-\gamma^{2} I, \\
& M_{3}=C^{T} D_{d}+P\left(B_{d}-H D_{d}\right), \\
& N_{1}=Q(A-H C)+(A-H C)^{T} Q-C^{T} C+\eta_{1} \rho^{2} I, \\
& N_{2}=-D_{f}^{T} D_{f}+\beta^{2} I, \\
& N_{3}=-C^{T} D_{f}+Q\left(B_{f}-H D_{f}\right) .
\end{aligned}
$$

Proof. By combining Theorems 6 and 7, we have Theorem 8 . This completes the proof.
Remark 9. Theorem 6 considers the robustness of residual signals to system uncertainty, and Theorem 7 considers the sensitivity of residual signals to system faults. Theorem 8 investigates the optimization of gain matrix $H$ by taking into account the robustness of residual signals to system uncertainty and sensitivity of residual signals to system faults simultaneously. If we iteratively use Theorem 8 , we can get the optimized solution of the performance indices $\gamma, \beta$ and gain matrix $H$ by using LMI toolbox in MatLab.

\section{Fault Detection Threshold}

In the above sections, we have investigated the optimization of the value of gain matrix $H$ in terms of LMIs. In this section, we will investigate the calculation of threshold for fault detection.

Theorem 10. Suppose Assumptions 1-3 hold. Consider the dynamic system described by (1), (2) and the FTA described by (4) (9); let the initial state and output of the FTA be $\hat{x}_{k}(0)=$ $x(0), \hat{y}_{k}(0)=y(0)(k=1,2, \ldots)$, respectively. If the following inequality holds

$$
\begin{aligned}
\left\|r_{k}(t)\right\|> & L_{w} P \sup _{t, \tau \in\left[t_{a}, t_{b}\right]}\left\|C \Phi(t, \tau)\left(B_{d}-H D_{d}\right)\right\| \\
+ & \left(\left(\sup _{t, \tau \in\left[t_{a}, t_{b}\right]}\|C \Phi(t, \tau)\| L_{w} P\right.\right. \\
& \left.\times \sup _{t, \tau \in\left[t_{a}, t_{b}\right]}\left\|\Phi(t, \tau)\left(B_{d}-H D_{d}\right)\right\|\right) \\
& \left.\times\left(\sup _{t, \tau \in\left[t_{a}, t_{b}\right]}\|\Phi(t, \tau)\|\right)^{-1}\right) \\
& \times \exp \rho \sup _{t, \tau \in\left[t_{a}, t_{b}\right]}\|\Phi(t, \tau)\| P
\end{aligned}
$$

then there is fault occurring in the system.

Proof. Without loss of generality, we assume $t \in\left[t_{a}, t_{b}\right]$ and $t_{b}-t_{a}=P$.

Subtracting (4) from system equation (1) and subtracting (5) from system equation (2) result in the estimation error dynamics:

$$
\begin{aligned}
\dot{e}(t)= & (A-H C) e(t)+\left(B_{f}-H D_{f}\right) f(t) \\
& +\left(B_{d}-H D_{d}\right) d(t)+g(x(t), t) \\
& -g(\widehat{x}(t), t)-B_{f} \widehat{f}(t), \\
r(t)= & C e(t)+D_{f} f(t)+D_{d} d(t) .
\end{aligned}
$$

In the condition of $\widehat{f}(t)=0$, we have

$$
\begin{aligned}
\dot{e}(t)= & (A-H C) e(t)+\left(B_{f}-H D_{f}\right) f(t) \\
& +\left(B_{d}-H D_{d}\right) d(t)+g(x(t), t)-g(\widehat{x}(t), t), \\
r(t)= & C e(t)+D_{f} f(t)+D_{d} d(t) .
\end{aligned}
$$


The solution of $(40)$ is $e_{k}(t)=\Phi\left(t, t_{a}\right) e_{k}\left(t_{a}\right)$

$$
\begin{aligned}
+\int_{t_{a}}^{t} \Phi(t, \tau)[ & \left(B_{f}-H D_{f}\right) f(\tau)+g_{k}(x(\tau), \tau) \\
& \left.-g_{k}(\widehat{x}(\tau), \tau)+\left(B_{d}-H D_{d}\right) d(\tau)\right] d \tau
\end{aligned}
$$

$$
\begin{aligned}
r_{k}(t)= & C \Phi\left(t, t_{a}\right) e_{k}\left(t_{a}\right) \\
+\int_{t_{a}}^{t} C \Phi(t, \tau) & {\left[\left(B_{f}-H D_{f}\right) f(\tau)+g_{k}(x(\tau), \tau)\right.} \\
& \left.\quad-g_{k}(\widehat{x}(\tau), \tau)+\left(B_{d}-H D_{d}\right) d(\tau)\right] d \tau
\end{aligned}
$$

where $\Phi(t)=L^{-1}\left[(s I-(A-H C))^{-1}\right] ; L^{-1}$ denotes inverse Laplacian transform.

Due to $\widehat{x}_{k}(0)=x(0), \widehat{y}_{k}(0)=y(0)(k=1,2, \ldots)$, we have $\widehat{y}_{k+1}\left(t_{a}\right)=C \hat{x}_{k+1}\left(t_{a}\right)=C \widehat{x}_{k}\left(t_{a}\right)=\widehat{y}_{k}\left(t_{a}\right), \quad r_{k}\left(t_{a}\right)=0$,

$$
e_{k}\left(t_{a}\right)=0 \text {. }
$$

Substituting (43) into (42) yields

$$
\begin{aligned}
r_{k}(t)=\int_{t_{a}}^{t} C \Phi(t, \tau)[ & \left(B_{f}-H D_{f}\right) f(\tau)+g_{k}(x(\tau), \tau) \\
& \left.-g_{k}(\widehat{x}(\tau), \tau)+\left(B_{d}-H D_{d}\right) d(\tau)\right] d \tau .
\end{aligned}
$$

According to Assumptions 1-3, the time weighted norm of (44) is

$$
\begin{aligned}
\left\|r_{k}(t)\right\| \leq & \int_{t_{a}}^{t}\left\|C \Phi(t, \tau)\left(B_{f}-H D_{f}\right) f(\tau)\right\| d \tau \\
& +\int_{t_{a}}^{t}\left\|C \Phi(t, \tau)\left(g_{k}(x(\tau), \tau)-g_{k}(\widehat{x}(\tau), \tau)\right)\right\| d \tau \\
& +\int_{t_{a}}^{t}\left\|C \Phi(t, \tau)\left(B_{d}-H D_{d}\right)\right\|\|d(\tau)\| d \tau .
\end{aligned}
$$

In the condition of $f(t)=0$, we have

$$
\begin{aligned}
\left\|r_{k}(t)\right\| \leq & \int_{t_{a}}^{t}\left\|C \Phi(t, \tau)\left(g_{k}(x(\tau), \tau)-g_{k}(\widehat{x}(\tau), \tau)\right)\right\| d \tau \\
& +\int_{t_{a}}^{t}\left\|C \Phi(t, \tau)\left(B_{d}-H D_{d}\right)\right\|\|d(\tau)\| d \tau .
\end{aligned}
$$

According to (41), we have

$$
\begin{aligned}
\left\|e_{k}(t)\right\| \leq & \int_{t_{a}}^{t} \rho\|\Phi(t, \tau)\|\left\|e_{k}(\tau)\right\| d \tau \\
& +\int_{t_{a}}^{t}\left\|\Phi(t, \tau)\left(B_{d}-H D_{d}\right)\right\|\|d(\tau)\| d \tau
\end{aligned}
$$

$$
\begin{aligned}
& \leq \int_{t_{a}}^{t} \rho\|\Phi(t, \tau)\|\left\|e_{k}(\tau)\right\| d \tau \\
& \quad+L_{w} P \sup _{t, \tau \in\left[t_{a}, t_{b}\right]}\left\|\Phi(t, \tau)\left(B_{d}-H D_{d}\right)\right\| .
\end{aligned}
$$

Using Gronwall-Bellman inequality, (47) can be simplified as

$$
\begin{aligned}
\left\|e_{k}(t)\right\| \leq & L_{w} P \sup _{t, \tau \in\left[t_{a}, t_{b}\right]}\left\|\Phi(t, \tau)\left(B_{d}-H D_{d}\right)\right\| \\
& \times \exp \rho \sup _{t, \tau \in\left[t_{a}, t_{b}\right]}\|\Phi(t, \tau)\|\left(t-t_{a}\right),
\end{aligned}
$$

where exp denotes exponential function. Substituting (48) into (46) yields

$$
\begin{aligned}
\left\|r_{k}(t)\right\| \leq & \int_{t_{a}}^{t}\left\|C \Phi(t, \tau)\left(g_{k}(x(\tau), \tau)-g_{k}(\hat{x}(\tau), \tau)\right)\right\| d \tau \\
& +\int_{t_{a}}^{t}\left\|C \Phi(t, \tau)\left(B_{d}-H D_{d}\right)\right\|\|d(\tau)\| d \tau \\
\leq & \int_{t_{a}}^{t} \rho\|C \Phi(t, \tau)\| \cdot\left\|e_{k}(t)\right\| d \tau \\
& +\int_{t_{a}}^{t}\left\|C \Phi(t, \tau)\left(B_{d}-H D_{d}\right)\right\|\|d(\tau)\| d \tau \\
\leq & L_{w} P \sup _{t, \tau \in\left[t_{a}, t_{b}\right]}\left\|C \Phi(t, \tau)\left(B_{d}-H D_{d}\right)\right\| \\
& +\left(\sup _{t, \tau \in\left[t_{a}, t_{b}\right]}\|C \Phi(t, \tau)\| L_{w} P\right. \\
& +\exp \sup _{t, \tau \in\left[t_{a}, t_{b}\right]}\|\Phi(t, \tau)\| P . \\
& \times L_{w} P \sup _{t, \tau \in\left[t_{a}, t_{b}\right]}\left\|C \Phi(t, \tau)\left(B_{d}-H D_{d}\right)\right\| \\
& \left.\quad \sup _{t, \tau \in\left[t_{a}, t_{b}\right]}\left\|\Phi(t, \tau)\left(B_{d}-H D_{d}\right)\right\|\right) \\
& \left.\times\left(\sup _{t, \tau \in\left[t_{a}, t_{b}\right]}\|C \Phi(t, \tau)\|\right)_{t, \tau \in\left[t_{a}, t_{b}\right]}\|\Phi(t, \tau)\|\right)
\end{aligned}
$$


If faults occur in the system, then

$$
\begin{aligned}
\left\|r_{k}(t)\right\|> & L_{w} P \sup _{t, \tau \in\left[t_{a}, t_{b}\right]}\left\|C \Phi(t, \tau)\left(B_{d}-H D_{d}\right)\right\| \\
+ & \left(\left(\sup _{t, \tau \in\left[t_{a}, t_{b}\right]}\|C \Phi(t, \tau)\| L_{w} P\right.\right. \\
& \left.\times \sup _{t, \tau \in\left[t_{a}, t_{b}\right]}\left\|\Phi(t, \tau)\left(B_{d}-H D_{d}\right)\right\|\right) \\
& \left.\times\left(\sup _{t, \tau \in\left[t_{a}, t_{b}\right]}\|\Phi(t, \tau)\|\right)^{-1}\right) \\
& \times \exp \rho \sup _{t, \tau \in\left[t_{d}, t_{b}\right]}\|\Phi(t, \tau)\| P .
\end{aligned}
$$

If the following inequality holds

$$
\begin{aligned}
\left\|r_{k}(t)\right\| \leq & L_{w} P \sup _{t, \tau \in\left[t_{a}, t_{b}\right]}\left\|C \Phi(t, \tau)\left(B_{d}-H D_{d}\right)\right\| \\
+ & \left(\left(\sup _{t, \tau \in\left[t_{a}, t_{b}\right]}\|C \Phi(t, \tau)\| L_{w} P\right.\right. \\
& \left.\times \sup _{t, \tau \in\left[t_{a}, t_{b}\right]}\left\|\Phi(t, \tau)\left(B_{d}-H D_{d}\right)\right\|\right) \\
& \left.\times\left(\sup _{t, \tau \in\left[t_{a}, t_{b}\right]}\|\Phi(t, \tau)\|\right)^{-1}\right) \\
& \times \exp \rho \sup _{t, \tau \in\left[t_{a}, t_{b}\right]}\|\Phi(t, \tau)\| P
\end{aligned}
$$

then there are no faults occurring in the system.

This completes the proof.

In the presence of unstructured modeling uncertainty, we have to determine the upper bounds of the residual signals for fault detection, referred to as the threshold. Theorem 10 investigated the calculation of threshold for fault detection. Once the residual signals exceed the threshold, it indicates that system faults occur. If the residual signal is below the threshold, there is no fault occurring. Moreover, we can use the residual evaluation function $\|r(t)\|_{2}$ to detect system faults $[20,21]$.

\section{Simulation Results}

In this section, we use the proposed method to detect faults for a class of uncertain nonlinear systems. Let us consider the uncertain nonlinear system with parameters as follows:

$$
\begin{aligned}
& \dot{x}(t)= {\left[\begin{array}{cc}
-0.38 & 0 \\
0 & -0.59
\end{array}\right] x(t)+\left[\begin{array}{l}
1 \\
1
\end{array}\right] u } \\
&+\left[\begin{array}{cc}
0.1 \sin (t) & 0.1 \sin (t)
\end{array}\right] x(t) \\
&+\left[\begin{array}{l}
0.2 \\
0.2
\end{array}\right] d(t)+\left[\begin{array}{l}
0.1 \\
0.1
\end{array}\right] f(t), \\
& y(t)=\left[\begin{array}{ll}
1 & 1
\end{array}\right] x(t)+0.1 f(t)+0.1 d(t) .
\end{aligned}
$$

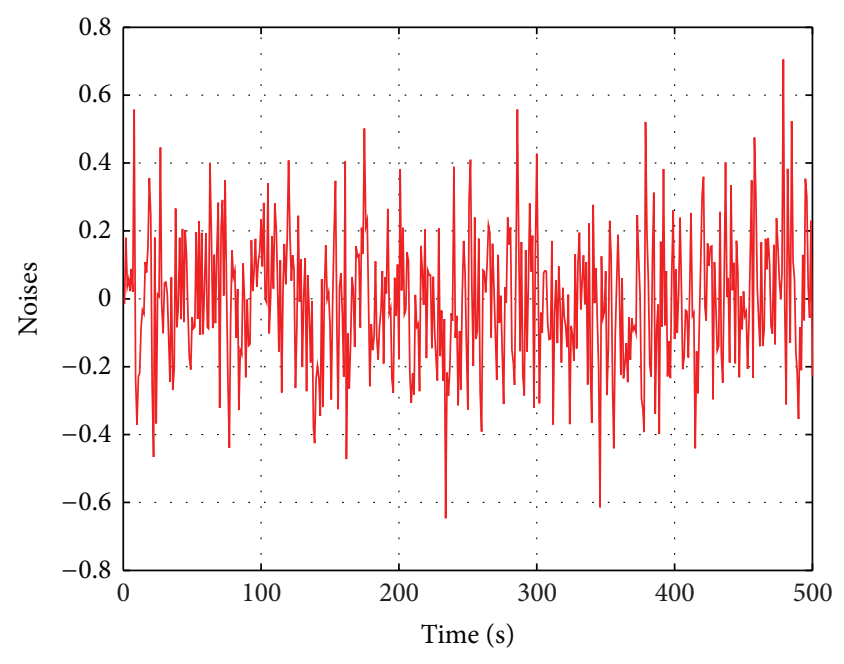

FIGURE 1: White noise signal.

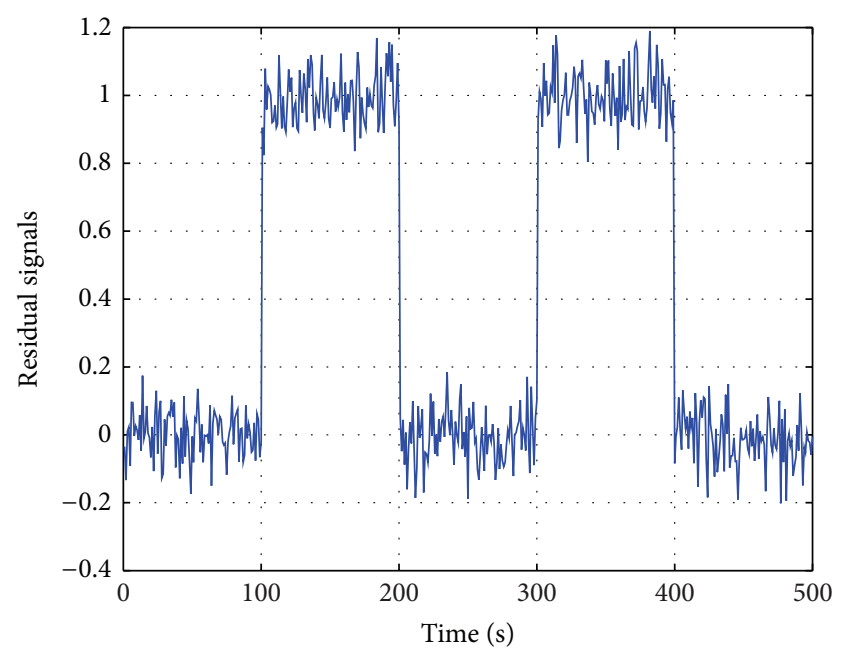

Figure 2: Generated residual signal.

Let $\gamma=0.3$, let $\beta=0.7$, and let $d(t)$ be white noise with energy 0.5 . According to Theorem 8 , the gain matrix of FTA can be determined: $H=[0.2071,0.1832]^{T}$. According to Theorem 10, the threshold for fault detection is 0.426 . The white noise signal, generated residual signal are shown in Figures 1 and 2, respectively.

From Figure 2, we can clearly see that the residual signal is below the threshold at time $t<100 \mathrm{~s}$. Therefore, there is no fault occurring at time $t<100 \mathrm{~s}$. At time $100 \mathrm{~s}<t<200 \mathrm{~s}$, the residual signal exceeds the threshold; it indicates that system fault occurs. Next, we use residual evaluation function $\|r(t)\|_{2}$ to detect system faults. Figure 3 shows the evolution of residual evaluation function $\|r(t)\|_{2}$. From Figure 3 we can see that, at time $100 \mathrm{~s}<t<200 \mathrm{~s}$, the residual evaluation function $\|r(t)\|_{2}$ jumps from 0.1 to 5.1 ; it indicates that system fault occurs. It can be seen from the simulation results that the proposed approach can improve not only the sensitivity of the FTA to system faults, but also the robustness of the FTA to systems uncertainty. 


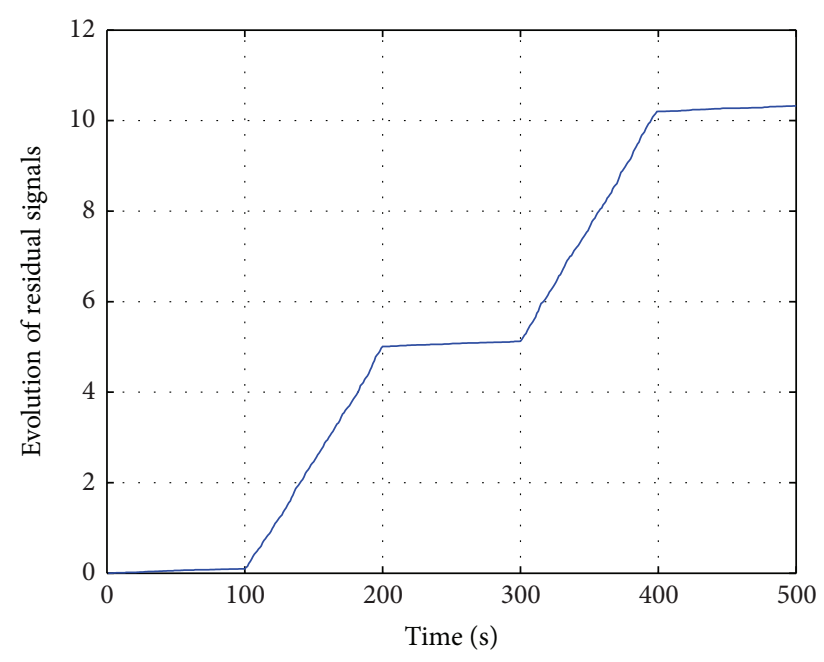

FIGURE 3: Evolution of residual evaluation function.

\section{Conclusions}

This paper has proposed a fault detection scheme for a class of uncertain nonlinear systems. The main contribution of this paper is to utilize robust control theory and multiobjective optimization algorithm to design the gain matrix of FTA. The gain matrix of FTA is designed to minimize the effects of system uncertainty on residual signals while maximizing the effects of system faults on residual signals. The calculation of the gain matrix is given in terms of LMIs formulations. The selection of threshold for fault detection is rigorously investigated as well. In the end, an illustrative example has demonstrated the validity and applicability of the proposed approach.

\section{Conflict of Interests}

The authors declare that there is no conflict of interests regarding the publication of this paper.

\section{Acknowledgments}

This work is supported by National Natural Science Foundation of China (nos. 51407078, 61201124) and Special Fund of East China University of Science and Technology for Basic Scientific Research (WJ1313004-1, WH1114027, H200-4-13192, and WH1414022).

\section{References}

[1] P. M. Frank, "Fault diagnosis in dynamic systems using analytical and knowledge-based redundancy-a survey and some new results," Automatica, vol. 26, no. 3, pp. 459-474, 1990.

[2] R. Isermann, "Process fault detection based on modeling and estimation methods: a survey," Automatica, vol. 20, no. 4, pp. 387-404, 1984.

[3] K. Zhang, B. Jiang, and V. Cocquempot, "Fast adaptive fault estimation and accommodation for nonlinear time-varying delay systems," Asian Journal of Control, vol. 11, no. 6, pp. 643$652,2009$.

[4] X. Zhang, T. Parisini, and M. M. Polycarpou, "Sensor bias fault isolation in a class of nonlinear systems," IEEE Transactions on Automatic Control, vol. 50, no. 3, pp. 370-376, 2005.

[5] A. T. Vemuri, "Sensor bias fault diagnosis in a class of nonlinear systems," IEEE Transactions on Automatic Control, vol. 46, no. 6, pp. 949-954, 2001.

[6] W. Zhang, X.-S. Cai, and Z.-Z. Han, "Robust stability criteria for systems with interval time-varying delay and nonlinear perturbations," Journal of Computational and Applied Mathematics, vol. 234, no. 1, pp. 174-180, 2010.

[7] L. Guo and H. Wang, "Fault detection and diagnosis for general stochastic systems using B-spline expansions and nonlinear filters," IEEE Transactions on Circuits and Systems. I. Regular Papers, vol. 52, no. 8, pp. 1644-1652, 2005.

[8] X. Zhang, M. M. Polycarpou, and T. Parisini, "Fault diagnosis of a class of nonlinear uncertain systems with Lipschitz nonlinearities using adaptive estimation," Automatica, vol. 46, no. 2, pp. 290-299, 2010.

[9] J. Fan and Y. Wang, "Fault detection and diagnosis of nonlinear non-Gaussian dynamic processes using kernel dynamic independent component analysis," Information Sciences, vol. 259, pp. 369-379, 2014.

[10] P. Tadić and Ž. Durović, "Particle filtering for sensor fault diagnosis and identification in nonlinear plants," Journal of Process Control, vol. 24, no. 4, pp. 401-409, 2014.

[11] W. Chen and M. Saif, "An iterative learning observer for fault detection and accommodation in nonlinear time-delay systems," International Journal of Robust and Nonlinear Control, vol. 16, no. 1, pp. 1-19, 2006.

[12] B. Yan, Z. Tian, and S. Shi, "A novel distributed approach to robust fault detection and identification," International Journal of Electrical Power \& Energy Systems, vol. 30, no. 5, pp. 343-360, 2008.

[13] W. Zhang, H. Su, H. Wang, and Z. Han, "Full-order and reduced-order observers for one-sided Lipschitz nonlinear systems using Riccati equations," Communications in Nonlinear Science and Numerical Simulation, vol. 17, no. 12, pp. 4968-4977, 2012.

[14] W. Zhang, H.-S. Su, Y. Liang, and Z.-Z. Han, "Non-linear observer design for one-sided Lipschitz systems: an linear matrix inequality approach," IET Control Theory and Applications, vol. 6, no. 9, pp. 1297-1303, 2012.

[15] W. Zhang, H. Su, F. Zhu, and D. Yue, "A note on observers for discrete-time lipschitz nonlinear systems," IEEE Transactions on Circuits and Systems II: Express Briefs, vol. 59, no. 2, pp. 123-127, 2012.

[16] X. Zhang, M. M. Polycarpou, and T. Parisini, "A robust detection and isolation scheme for abrupt and incipient faults in nonlinear systems," IEEE Transactions on Automatic Control, vol. 47, no. 4, pp. 576-593, 2002.

[17] Y. Bingyong, T. Zuohua, and L. Dongmei, "Fault diagnosis for a class of nonlinear stochastic time-delay systems," Control and Instruments in Chemical Industry, vol. 34, no. 1, pp. 12-15, 2007.

[18] M. Zhong, S. X. Ding, J. Lam, and H. Wang, "An LMI approach to design robust fault detection filter for uncertain LTI systems," Automatica, vol. 39, no. 3, pp. 543-550, 2003.

[19] S. Xie, S. Tian, and Y. Fu, "A new algorithm of iterative learning control with forgetting factors," in Proceedings of the 8th Control, Automation, Robotics and Vision Conference (ICARCV'04), vol. 1, pp. 625-630, 2004. 
[20] H. Yang and M. Saif, "State observation, failure detection and isolation (FDI) in bilinear systems," International Journal of Control, vol. 67, no. 6, pp. 901-920, 1997.

[21] D. Yu and D. N. Shields, "A bilinear fault detection filter," International Journal of Control, vol. 68, no. 3, pp. 417-430, 1997. 


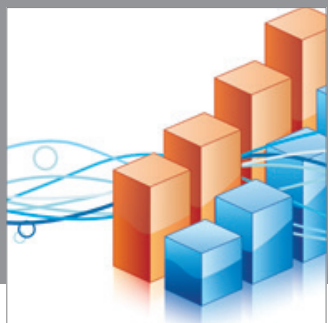

Advances in

Operations Research

mansans

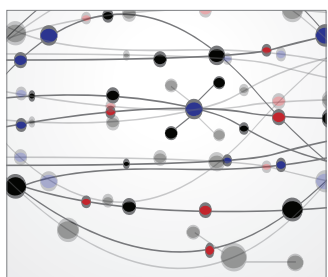

The Scientific World Journal
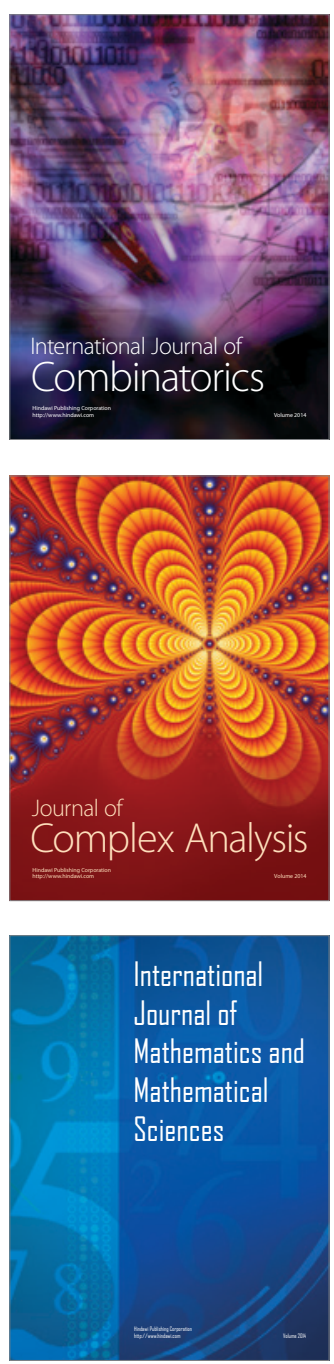
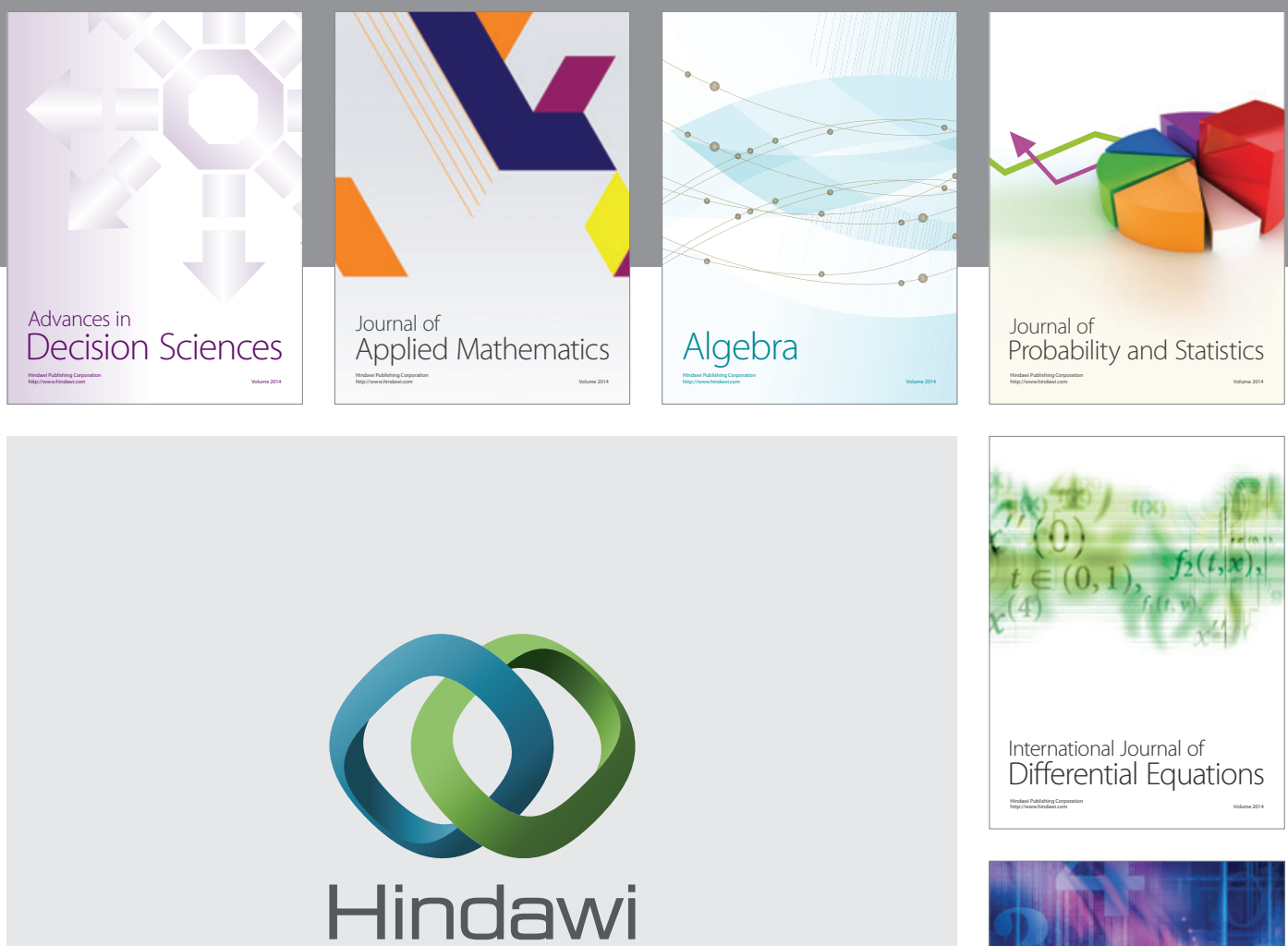

Submit your manuscripts at http://www.hindawi.com
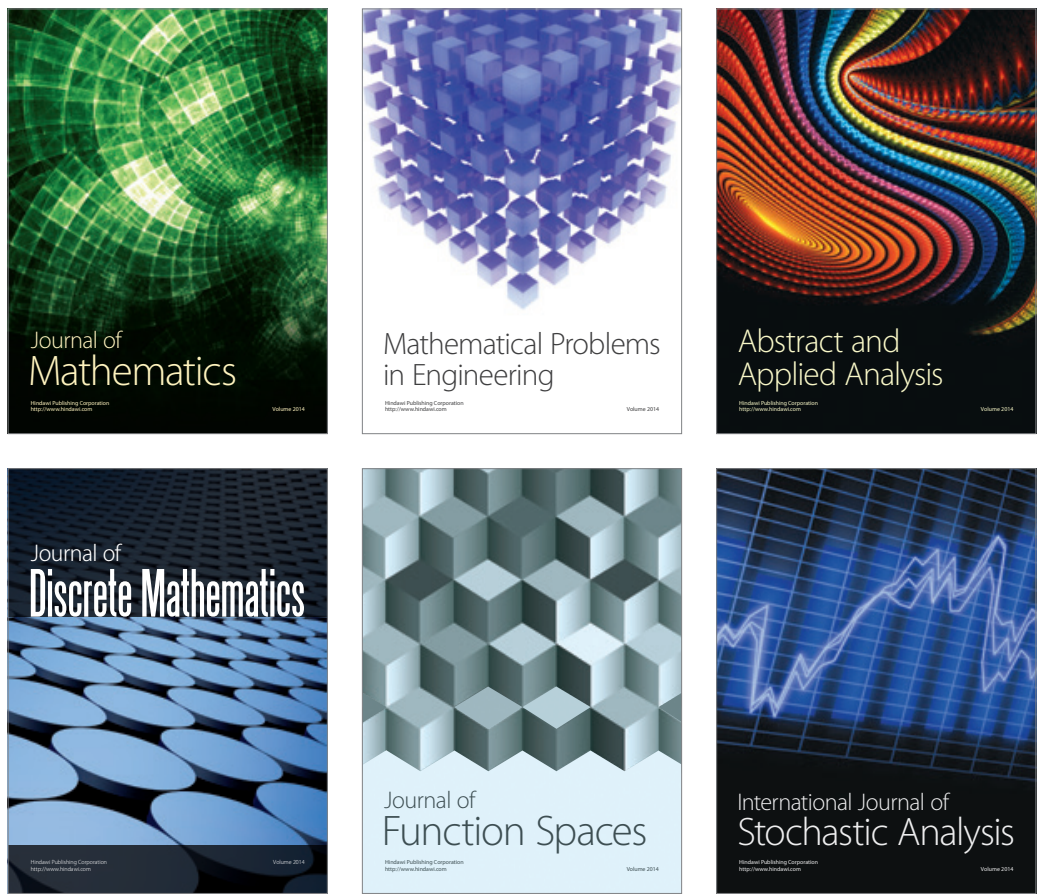

Journal of

Function Spaces

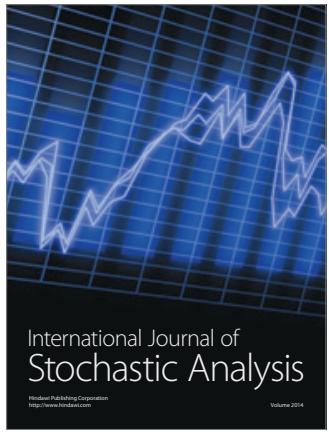

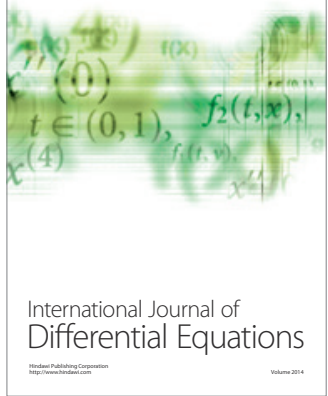
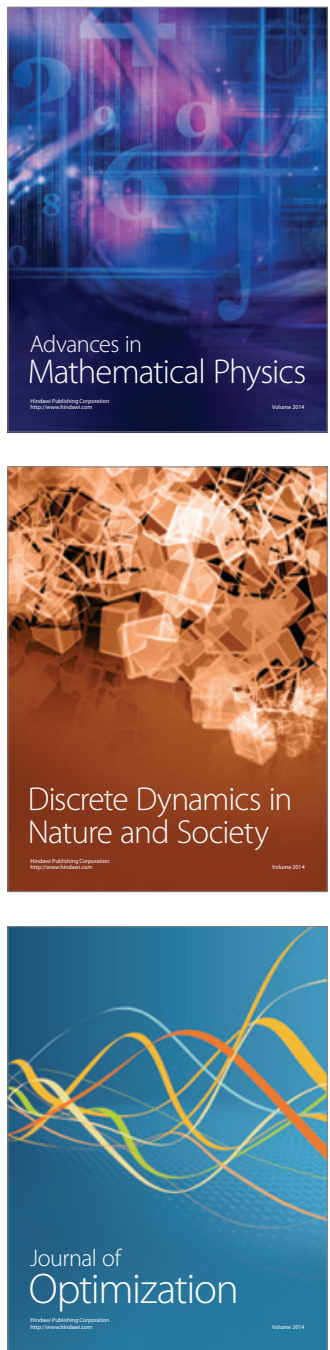